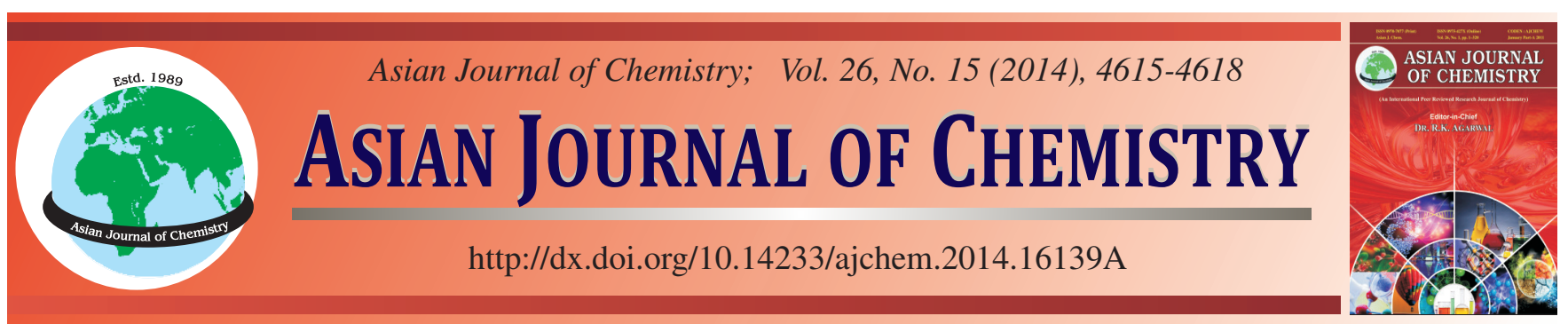

\title{
Optimization of Ethanol Extraction Process of Solanum nigrum Linn. and Structural Confirmation of its Compounds
}

\author{
SHAN-Bin GUO ${ }^{1}$, Yue Tian ${ }^{2}$ and Ling-YAN JiAN ${ }^{1, *}$
}

${ }^{1}$ Department of Pharmacy, Shengjing Hospital of China Medical University, Shenyang, P.R. China

${ }^{2}$ Department of Anesthesiology, Shengjing Hospital of China Medical University, Shenyang, P.R. China

*Corresponding author: Tel: +86 24966157 1101; E-mail: jianlingyan126@ 126.com

\begin{abstract}
This work reported the optimization of the ethanolic extraction process of Solanum nigrum Linn. and isolation, purification and identification of alkaloid constituents in Solanum nigrum Linn.. Orthogonal design was used to study the process optimization conditions, repeated silica gel column chromatography, preparative TLC, ODS column chromatography, Sephadex LH-20 column chromatography and preparative HPLC were used to isolate the compounds. It can be seen from the results of orthogonal experiment and variance analysis that, the optimal extraction process of Solanum nigrum Linn. with solasonine yield as investigation index is A3D3B3C3, i.e. addition of a 10fold amount of $90 \%$ ethanol, three times of extraction and extraction duration of $3 \mathrm{~h}$ each; four compounds, namely solamargine, solasonine, uttroside A and uttroside B, were isolated by subjecting the alkaloid fraction of Solanum nigrum Linn. to repeated silica gel column chromatography, preparative TLC, ODS column chromatography, Sephadex LH-20 column chromatography and preparative HPLC. $90 \%$ ethanol can be used in the extraction of Solanum alkaloids, Solanum nigrum Linn. contains constituents such as solamargine, solasonine, uttroside A and uttroside B.
\end{abstract}

Keywords: Solanum nigrum Linn, Orthogonal design, Solamargine, Uttroside A.

\section{INTRODUCTION}

Long Kui is the dried whole plant of Solanum nigrum Linn. or S. photeinocarpum Nakamura et Odashima, which is also named as Ye Pu Tao, Lao Ya Yan Jing Cao, Ku Cai, Ku Kui. It is annual to perennial herbaceous plant and is distributed throughout China ${ }^{1}$. The whole plant can be used as medicine, which is a common Chinese herbal medicine. Domestic and foreign scholars have conducted a lot of researches on Long Kui. So far, it has been confirmed that the whole plant and fruit of Long Kui contain a variety of chemical constituents, which mainly include three categories: alkaloids ${ }^{2-3}$, saponins and non-saponin constituents ${ }^{4}$.

Modern pharmacological studies have demonstrated that Long Kui has antitumor ${ }^{5-7}$, antiinflammatory ${ }^{8}$, antishock, antiallergic, heat-clearing, detoxifying, expectorant, antitussive and hypotensive ${ }^{9-11}$ effects. In this paper, the ethanol extraction process conditions of Solanum nigrum Linn. were optimized and the effects of factors such as different ethanol concentration, extraction duration, extraction times and ethanol amount on the yield of Solanum nigrum Linn. were determined with solasonine as an index. In the meanwhile, chemical constituents in Solanum nigrum Linn. were explored.

\section{EXPERIMENTAL}

Agilent 1200 HPLC (Agilent, USA); Bruker Avance 600 NMR spectrometer, X-6 micro melting point apparatus, Sephadex LH-20 (Pharmacia Bioteck).

Long Kui, collected from the surroundings of Shenyang, which was identified as the whole plant of Solanum nigrum Linn. in the genus Solanum of the family Solanaceae. Solasonine, prepared by our laboratory, purity of $97.4 \%$. Solvents were of analytical and chromatographic grades.

Chromatographic conditions: Column: Agilent Zorbax SB C18 column. Mobile phase: acetonitrile (A)-2 \% phosphoric acid solution (B) (volume ratio of 22:78). Detection wavelength: $205 \mathrm{~nm}$, flow rate: $1 \mathrm{~mL} / \mathrm{min}$; sample loading volume: $20 \mu \mathrm{L}$.

Preparation of standard curves: $5 \mathrm{mg}$ of solasonine reference substance was accurately weighed, placed in a $5 \mathrm{~mL}$ volumetric flask, dissolved by addition of mobile phase and diluted to the mark, then shaken uniformly to prepare a 1.125 $\mathrm{mg} / \mathrm{mL}$ reference solution; $0.3,0.7,1.1,1.5,1.9,2.3$ and 2.7 $\mathrm{mL}$ of the solution were precisely aspirated and placed in 5 $\mathrm{mL}$ volumetric flasks, respectively and the volume was made constant; $20 \mu \mathrm{L}$ of the above reference solutions were aspirated, 
respectively and subjected to HPLC according to the above conditions, peak areas were recorded; linear regression was performed with content $(\mathrm{Lg})$ as the abscissa $(\mathrm{X})$ and peak area as the ordinate $(\mathrm{Y})$ to obtain the equation $\mathrm{Y}=256483 \mathrm{X}+$ $19.746, r=0.9996$, which indicated that the linear relationship was good within the experimental range.

Preparation of samples: $50 \mathrm{~g}$ of medicinal material was accurately weighed in nine copies, sample extracts were prepared according to the L9(34) orthogonal table, then evaporated to dryness under reduced pressure, dissolved in $10 \%$ acetic acid solution, volume was made up to the mark in $10 \mathrm{~mL}$ volumetric flasks and solasonine content was determined.

Orthogonal experimental design: According to the pretest results, ethanol amount, ethanol concentration, extraction time (h) and extraction times had relatively great impacts on the extraction yield, so orthogonal experimental method was adopted and three levels were selected for each factor, factors and levels are shown in Table-1.

\section{TABLE-1}

FACTORS AND LEVELS OF ORTHOGONAL EXPERIMENT

\begin{tabular}{ccccc}
\hline Level & $\begin{array}{c}\text { A Ethanol } \\
\text { amount } \\
\text { (multiples) }\end{array}$ & $\begin{array}{c}\text { B Ethanol } \\
\text { concentration } \\
(\%)\end{array}$ & $\begin{array}{c}\mathrm{C} \\
\text { Extraction } \\
\text { time (h) }\end{array}$ & $\begin{array}{c}\text { Extraction } \\
\text { times } \\
\text { (times) }\end{array}$ \\
\hline 1 & 6 & 40 & 1 & 1 \\
2 & 8 & 60 & 2 & 2 \\
3 & 10 & 90 & 3 & 3 \\
\hline
\end{tabular}

Extraction and isolation of Solanum nigrum Linn: $5 \mathrm{~kg}$ of the whole plant of Solanum nigrum Linn. was taken, dried, cut into pieces about $5 \mathrm{~cm}$ long and extracted three times by heat reflux extraction with a 10 -fold amount of $90 \%$ ethanol, each extraction lasted $3 \mathrm{~h}$, then the extracts were combined and concentrated under reduced pressure to give the crude extract. The extract was added with an appropriate amount of water and mixed well, then isolated by resin column (D-101 macroporous adsorption resin column), eluted separately with water, $20 \%$ ethanol, $40 \%$ ethanol, $60 \%$ ethanol, $95 \%$ ethanol and anhydrous ethanol and identified by TLC with $500 \mathrm{~mL}$ as one fraction, then the identical fractions were combined to give Solanum alkaloid crude extract. The total alkaloid fraction of Solanum nigrum Linn. was subjected to repeated silica gel column chromatography, preparative TLC, ODS column chromatography, Sephadex LH-20 column chromatography and preparative HPLC to isolate four compounds.

\section{RESULTS AND DISCUSSION}

Optimization results of Solanum nigrum Linn. extraction process: In this experiment, orthogonal optimization was performed according to the orthogonal table with the yield of solasonine as the investigation index and analysis of variance was performed on solasonine extraction yield, the results are shown in Tables 2 and 3.

Range (R) analysis with the extraction yield of solasonine as the investigation index showed that the factors influencing the extraction efficiency of Solanum nigrum Linn. were D > $\mathrm{A}>\mathrm{B}>\mathrm{C}$ in descending order; analysis of variance results showed that: factor $\mathrm{A}$ and factor $\mathrm{D}$ had considerable significances on the yield of solasonine, while factor B and factor $\mathrm{C}$ had smaller impacts. It can be seen from the results of orthogonal
TABLE-2

OPTIMIZATION RESULTS OF Solanum nigrum LINN. EXTRACTION PROCESS

\begin{tabular}{cccccc}
\hline $\begin{array}{c}\text { Experiment } \\
\text { No. }\end{array}$ & $\mathrm{A}$ & $\mathrm{B}$ & $\mathrm{C}$ & $\mathrm{D}$ & $\begin{array}{c}\text { Solasonin } \\
\text { yield }(\mathrm{mg} / \mathrm{g})\end{array}$ \\
\hline 1 & 1 & 1 & 1 & 1 & 0.063 \\
2 & 1 & 2 & 2 & 2 & 0.144 \\
3 & 1 & 3 & 3 & 3 & 0.252 \\
4 & 2 & 1 & 2 & 3 & 0.318 \\
5 & 2 & 2 & 3 & 1 & 0.187 \\
6 & 2 & 3 & 1 & 2 & 0.242 \\
7 & 3 & 1 & 3 & 2 & 0.322 \\
8 & 3 & 2 & 1 & 3 & 0.331 \\
9 & 3 & 3 & 2 & 1 & 0.236 \\
\hline $\mathrm{k}_{1}$ & 0.459 & 0.702 & 0.636 & 0.486 & \\
$\mathrm{k}_{2}$ & 0.747 & 0.663 & 0.699 & 0.708 & \\
$\mathrm{k}_{3}$ & 0.888 & 0.729 & 0.762 & 0.900 & \\
$\mathrm{~K}_{1}$ & 0.153 & 0.234 & 0.212 & 0.162 & \\
$\mathrm{~K}_{2}$ & 0.249 & 0.221 & 0.233 & 0.236 & \\
$\mathrm{~K}_{3}$ & 0.296 & 0.243 & 0.254 & 0.300 & \\
$\mathrm{R}$ & 0.107 & 0.022 & 0.042 & 0.138 & \\
\hline
\end{tabular}

TABLE-3

ANALYSIS OF VARIANCE OF SOLASONINE YIELD

\begin{tabular}{lccccc}
\hline Factor & $\begin{array}{l}\text { Sum of } \\
\text { squared } \\
\text { deviations }\end{array}$ & $\begin{array}{l}\text { Degree } \\
\text { of } \\
\text { freedom }\end{array}$ & $\begin{array}{l}\mathrm{F} \\
\text { ratio }\end{array}$ & $\begin{array}{l}\mathrm{F} \\
\text { critical } \\
\text { value }\end{array}$ & $\begin{array}{l}\text { Signi- } \\
\text { ficance }\end{array}$ \\
\hline Ethanol amount & 0.032 & 2 & 32 & 19 & $*$ \\
$\begin{array}{l}\text { Ethanol } \\
\text { concentration }\end{array}$ & 0.001 & 2 & 1 & 19 & \\
Extraction time & 0.003 & 2 & 3 & 19 & $*$ \\
Extraction times & 0.029 & 2 & 29 & 19 & $*$ \\
Error & 0 & 2 & & & \\
\hline
\end{tabular}

experiment and variance analysis that the optimal extraction process of Solanum nigrum Linn. with extraction yield of solasonine as investigation index was $\mathrm{A} 3 \mathrm{D} 3 \mathrm{~B} 3 \mathrm{C} 3$, i.e. addition of a 10 -fold amount of $90 \%$ ethanol, three times of extraction and extraction duration of $3 \mathrm{~h}$ each.

Structural confirmation of compounds: Compound $\mathbf{1}$, Molisch reaction positive and bismuth potassium iodide staining positive, suggesting the possibility of alkaloid saponins.

Characteristic signals in the ${ }^{1} \mathrm{H}$ NMR spectrum: an alkenyl hydrogen proton signal s $5.35(1 \mathrm{H}$, br.d, $J=5.2 \mathrm{~Hz}, \mathrm{H}-6)$ and a 3-carbon end-group proton signal s 4.92 (Glc-1'), 5.83 (Rha1", br.s), 6.35 (Rha-1", br.s) appeared in the low field. High field region showed four characteristic methyl peaks of steroid nucleus.

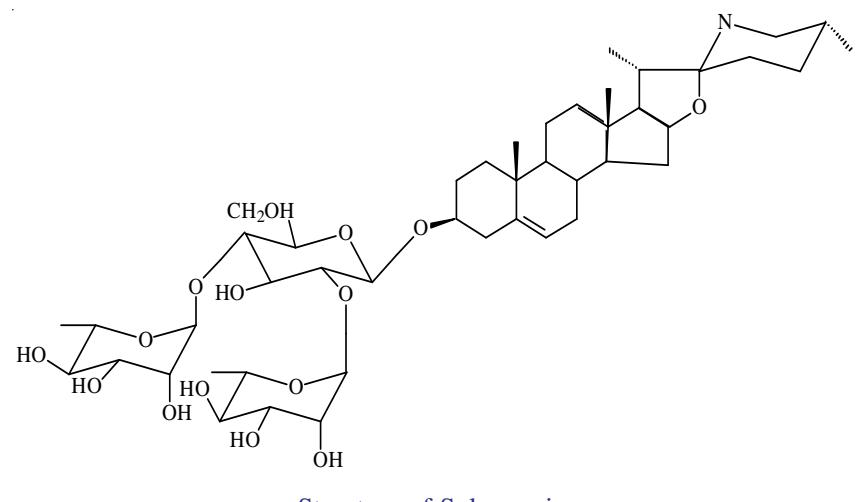

Structure of Solamargine 
The above data were basically consistent with the literature $^{12}$, so the structure of compound $\mathbf{1}$ was solamargine.

Compound 2, white powder, Molisch reaction positive, suggesting the possibility of alkaloid saponins.

In the ${ }^{1} \mathrm{H}$ NMR spectrum, an alkenyl hydrogen proton signal s 5.35 ( $1 \mathrm{H}$, br.d, $J=5.2 \mathrm{~Hz}, \mathrm{H}-6$ ) and a 3-carbon endgroup proton signal s $4.96(\mathrm{Gal}-1$ ', d, $J=7.5 \mathrm{~Hz}), 5.14$ (Glc-1 ", d, $J=7.8 \mathrm{~Hz}$ ), 6.23 (Rha-1"', br.s) appeared in the low field. In the high field region, four characteristic methyl peaks of steroid nucleus s $0.87(3 \mathrm{H}, \mathrm{s}, \mathrm{Me}-18), 1.12(3 \mathrm{H}, \mathrm{s}$, Me-19), 1.14 (3H, d, $J=7.0 \mathrm{~Hz}, \mathrm{Me}-21), 0.87$ (3H, d, $J=5.2$ $\mathrm{Hz}, \mathrm{Me}-27$ ), as well as the Rha-6"'-Me signal s 1.69 (d, $J=6.2$ $\mathrm{Hz}$ ) were shown.

The above data were basically consistent with the literature $^{12}$, so the structure of compound $\mathbf{2}$ was solasonine.

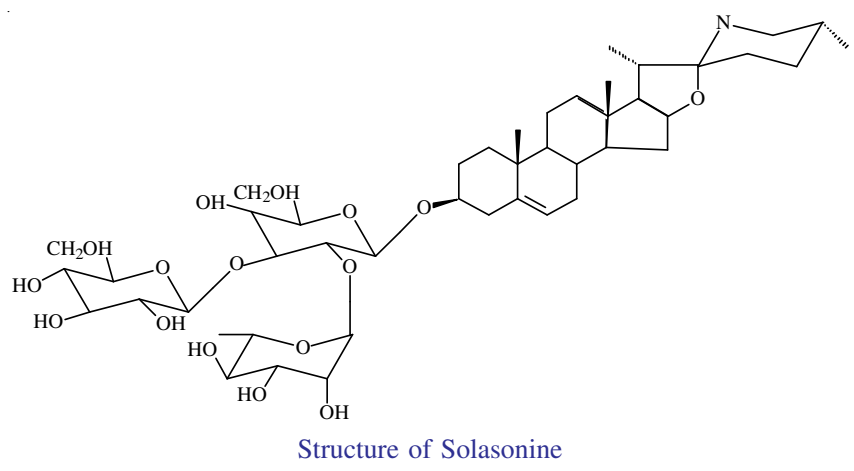

Compound 3, white powder. Liebermann-Burchard and Molisch reactions positive.

The above data were basically consistent with the literatures $^{13,14}$, so the structure of compound 3 was uttroside $\mathrm{B}$, i.e. (22 $\alpha, 25 \mathrm{R})-26-\mathrm{O}-\beta$-D-glucopyranosyl-22-hydroxyl-5 $\alpha$ furost-3 $\beta$, 26-diol-3-O- $\beta$-D-glucopyranosyl-(1-2)-O-[ $\beta-3-D-$ xylopyranosyl-(1-3)]-O- $\beta$-D-glucopyranosyl-(1-4)-O- $\beta$-Dgalactopyranoside.

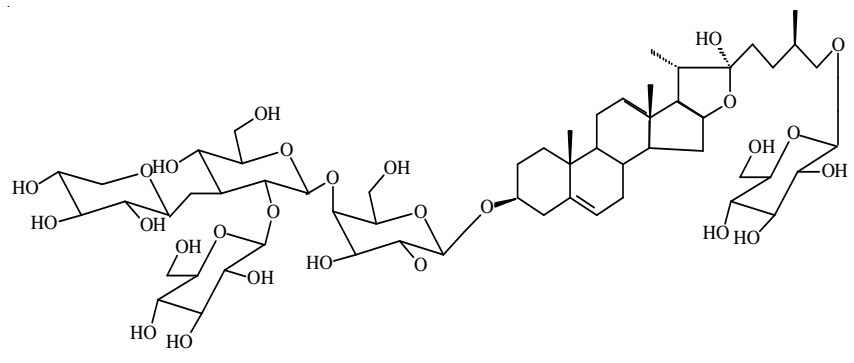

Structure of Uttroside B

Compound 4, white amorphous powder. LiebermannBurchard and Molisch reactions positive, stained pink with the Ehrlich's reagent, suggesting that the compound was a furostanol saponin.

The ${ }^{1} \mathrm{H}$ NMR and ${ }^{13} \mathrm{C}$ NMR data of compound 4 were very similar to compound $\mathbf{3}$, with the exceptions of an additional oxymethyl signal $(\delta 3.67, \mathrm{~s})$ in the ${ }^{1} \mathrm{H}$ NMR spectrum and an additional carbon signal at $\delta 47.6$. In addition, compared with compound $\mathbf{3}$, the $22^{\text {nd }}$ bit of compound $\mathbf{4}$ had a $\delta 2.7$ displacement toward low field, suggesting that compound 4 was the 22-methoxylation product of compound $\mathbf{3}$, through comprehensive analysis, compound $\mathbf{4}$ was identified as uttroside $\mathrm{A}$, i.e. $(22 \alpha, 25 \mathrm{R})-26-\mathrm{O}-\beta-\mathrm{D}$-glucopyranosyl-22methoxy-5 $\alpha$-furost-3 $\beta$, 26-diol-3-O- $\beta$-D-glucopyranosyl-(12)-O-[ $\beta$-D-xylopyranosyl-(1-3)]-O- $\beta$-D-glucopyranosyl-(14)-O- $\beta$-D-galactopyranoside.

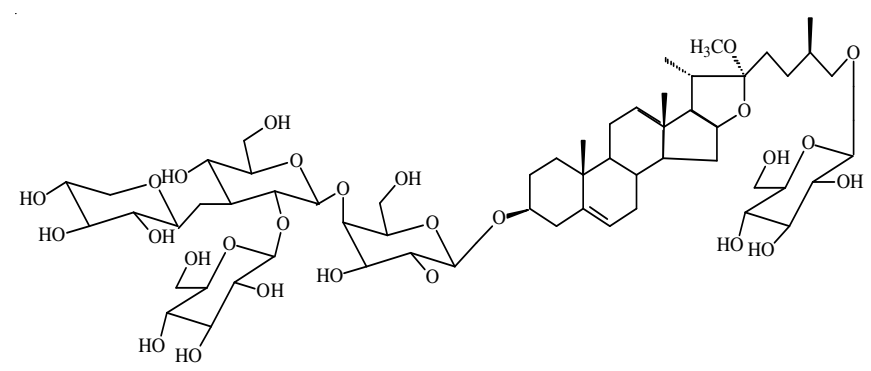

Structure of Uttroside A

Most of the Solanum alkaloids are water-soluble alkaloids ${ }^{15}$. Conventional alkaloid extraction methods include water or acid water extraction, ethanol solvent extraction, ion exchange resin method, etc. Due to the larger volume of water or acidic water used in the water or acid water extraction process, removal and concentration are time- and energyconsuming and subsequent processing is relatively difficult. Alkaloids and their salts are generally easily dissolved in ethanol, so the extraction of Solanum alkaloids using ethanol as a solvent is theoretically well grounded.

In this experiment, on the basis of the preliminary experiment, ethanol was selected as the extraction solvent for Solanum alkaloids, process optimization is performed on important influential factors such as concentration and amount of ethanol, duration and times of extraction using orthogonal experimental method, it can be seen from the results of orthogonal experiment and variance analysis that the optimal extraction process of Solanum nigrum Linn. with solasonine yield as investigation index was $\mathrm{A} 3 \mathrm{D} 3 \mathrm{~B} 3 \mathrm{C} 3$, i.e. addition of a 10 -fold amount of $90 \%$ ethanol, three times of extraction and extraction duration of $3 \mathrm{~h}$ each.

Steroid alkaloids contained in Solanum plants are a class of steroidal structure containing alkaloids, which are regarded as the simple nitrogenous derivatives of natural steroids and collectively referred to as pseudo-alkaloids together with terpenoid alkaloids. The structure of steroid alkaloids is relatively complex, which specifically belongs to the cholestane derivatives in the steroidal alkaloids. Among them, the representative are solasonine and solamargine, where the content of the latter is higher than the former. Several scholars have done relatively many researches on the chemical constituents of Solanum species and isolated some compounds from them $^{16,17}$. In this paper, crude extract of Solanum nigrum total alkaloids is obtained by optimizing the ethanol extraction process conditions of Solanum nigrum Linn.

Four compounds are isolated by subjecting the total alkaloid fraction of Solanum nigrum Linn. to repeated silica gel column chromatography, preparative TLC, ODS column chromatography, Sephadex LH-20 column chromatography and preparative HPLC, which are solamargine, solasonine, uttroside A and uttroside B, respectively. The structures of these compounds are well eludicated. 


\section{REFERENCES}

1. R.-M. Lu, X.-W. Tan and Y.Y. Zhou, Lishizhen Med. Mater. Med. Res., 1821 (2009)

2. M.-H. Li, G. Ding and Z.-Q. Meng, Chinese J. Nat. Med., 360 (2007).

3. S. Sadasivam and E.R. Shanmugasundaram, Enzymologia, 31, 203 (1966).

4. $\quad$ K. Larsen, Nord. Med., 66, 1418 (1961).

5. Y.-B. Ji, H.-L. Wang and S.-Y. Gao, Chin. Tradit. Herbal Drugs, 1200 (2005).

6. T. Ikeda, H. Tsumagari, T. Honbu and T. Nohara, Biol. Pharm. Bull., 26, 1198 (2003).

7. S.-H. Wang, Y.-F. Cong, M. Liang and S.Q. Wang, Heilongjiang Medical J., 421 (2005).

8. Y.O. Son, J. Kim, J.C. Lim, Y. Chung, G.-H. Chung and J.-C. Lee, Food Chem. Toxicol., 41, 1421 (2003).
9. S. Sultana, S. Perwaiz, M. Iqbal and M. Athar, J. Ethnopharmacol., 45, 189 (1995).

10. K. Raju, G. Anbuganapathi, V. Gokulakrishnan, B. Rajkapoor, B. Jayakar and S. Manian, Biol. Pharm. Bull., 26, 1618 (2003).

11. J. He, H.M. Shen and X.-H. Zhang, J. Gansu Agric. Univ., 447 (2004).

12. S.B. Mahato, N.P. Sahu, A.N. Ganguly, R. Kasai and O. Tanaka, Phytochemistry, 19, 2017 (1980).

13. X.H. Zhu, H. Tsumagari, T. Honbu, T. Ikeda, M. Ono and T. Nohara, Tetrahedron Lett., 42, 8043 (2001).

14. J.M. Jin, Y.J. Zhang and C.R. Yang, J. Nat. Prod., 67, 5 (2004).

15. L.-Y. Wang, N.L.Wang and X.-S. Yao, J. Chin. Med. Mater., 30, 792 (2007).

16. S.M. Aslanov, Khim. Prir. Soedin., 5, 674 (1971).

17. H. Ripperger, Phytochemistry, 39, 1475 (1995). 